\title{
Engagement of Accountable Care Organizations in Acute Care Redesign: Results of a National Survey
}

\author{
Michelle P. Lin, MD, MPH, MS ${ }^{7}$, David Muhlestein, PhD, JD², Brendan G. Carr, MD, MA, MS , \\ Lynne D. Richardson, $M D^{7}$, Jennifer L. Wiler, MD, MBA ${ }^{4}$, and Jeremiah D. Schuur, MD, $M H S^{5}$ \\ 'Department of Emergency Medicine, Icahn School of Medicine at Mount Sinai, New York, NY, USA; ${ }^{2}$ Leavitt Partners, Salt Lake City, UT, USA; \\ ${ }^{3}$ Sidney Kimmel Medical College, Thomas Jefferson University, Philadelphia, PA, USA; ${ }^{4}$ University of Colorado School of Medicine, Denver, CO, USA; \\ ${ }^{5}$ Brigham and Women's Hospital and Harvard Medical School, Boston, MA, USA.
}

KEY WORDS: health services; accountable care; healthcare Reform.

J Gen Intern Med 33(10):1601-3

DOI: $10.1007 / \mathrm{s} 11606-018-4525-4$

(c) Society of General Internal Medicine 2018

\section{INTRODUCTION}

Accountable Care Organizations (ACOs) are integrated provider groups aiming to improve health care value for a population. Hospitalizations account for $\sim 32 \%$ of US health care expenditures, and ED visits account for up to $6 \%$; therefore, one strategy to improve value is to manage the acute care needs of the population in lower cost settings. ${ }^{1}$ To date, most cost-reduction efforts have focused on improving chronic disease management, for example, through medication management and decreasing readmissions. ${ }^{2}$

We aim to describe how ACOs are prioritizing costreduction strategies related to acute unscheduled care using responses from a national survey of ACOs.

\section{METHODS}

We surveyed all known ACOs in the USA from January 2017 to April 2017; these included participants in Medicare, commercial, and Medicaid insurance programs. We asked ACO leaders to prioritize six strategies to address acute unscheduled care (including ED and urgent care) including 4 types of care redesign, and 2 types of measures or incentives, which are listed in Figures 1 and 2. Prioritization used a 5-point Likert scale from "very high priority" (5) to "very low priority" (1). We developed the survey in collaboration with emergency physicians and health service researchers with experience in survey methodology.

We collapsed responses into three categories: high, medium, and low priority. We analyzed proportions and chi-square $p$-values of stratified analyses by ACO variables, including ownership, region, number of covered lives, and any payer risk-sharing.

Published online June 8, 2018

\section{RESULTS}

The national ACO survey achieved a response rate of $27.4 \%$ (240/875); respondents were more likely than non-respondents to be Medicare ACOs, but otherwise similar with respect to region, size, and organizational structure. The strategies to generate value most frequently ranked highly by ACOs were primary care redesign $(59.2 \%$, or $142 / 240)$, and creating alternative sites to the ED for acute unscheduled care (e.g., urgent care) $(52.1 \%$, or $125 / 240)$ (Fig. 1). The two lowest ranked priorities were expanding alternatives to inpatient hospitalization for ED patients (e.g., observation units) $(40.4 \%$, or $97 / 240)$ and the development of measures or incentives for ED providers to reduce inpatient hospitalizations $(34.2 \%$, or $82 / 240)$. ACOs engaged in any risk sharing were more likely to place high priority on primary care redesign $(P=0.041)$, creating alternative acute care sites $(P=0.007)$, and expanding alternatives to inpatient hospitalization $(P=0.017)$ than those ACOs not taking risk. ACOs that ranked creating alternatives to the ED highly were more likely to have a larger number of covered lives $(P=0.006)$ (Fig. 2). ACOs that ranked expanding alternatives to inpatient hospitalization for ED patients highly were more likely to be in the Northeast $(P=0.006)$.

\section{DISCUSSION}

ACOs prioritize strategies to reduce ED visits above reducing potentially avoidable inpatient admissions from the ED, despite significantly larger health care expenditures associated with inpatient hospitalizations. ACOs placed the highest priority on primary care redesign and alternatives to the ED to reduce costs associated with acute unscheduled care. While interventions to reduce ED use through primary care redesign such as increased after-hours access and patient-centered medical homes have shown limited success, it remains unclear whether the substantial investments required to enhance primary care infrastructure as a means of reducing acute care utilization are cost-effective. ${ }^{3,4}$ Furthermore, recent reviews have highlighted 


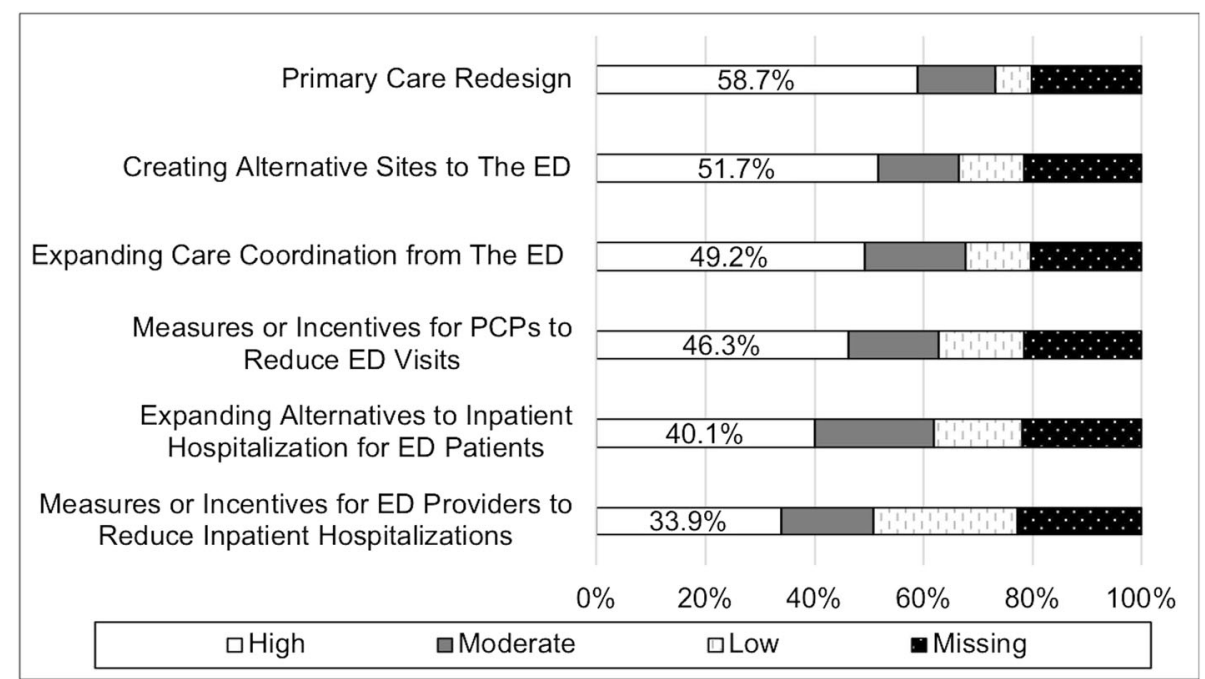

Fig. 1 ACO priorities for acute unscheduled care redesign.

the dearth of high-quality evidence on the effectiveness of ED visit reduction programs. ${ }^{5}$

ACOs choice to prioritize financial incentives for primary care physicians (PCPs) to reduce ED visits over incentives for ED clinicians to reduce hospitalizations warrants review. Hospitalizations are more costly than ED visits, representing one third of ACO's spending, and studies suggest high-performing Medicare ACOs achieved greater reductions in hospital inpatient expenditures relative to other services. ${ }^{6}$ Furthermore, ED providers have more control over decisions to admit patients to the hospital than PCPs have over a patient's decision to seek care in the ED. Significant opportunities exist to expand on the role of emergency care in value-based health care by promoting outpatient pathways after ED evaluation, including expedited outpatient follow-up and transitional care, observation care, home-based care, and hospitalization at home.

Limitations of this study include the survey response rate, although the number of respondents is comparable or high relative to prior surveys of ACOs and is nationally representative, as well as the self-reported nature of responses, which may not be associated with actual strategies implemented within ACOs.

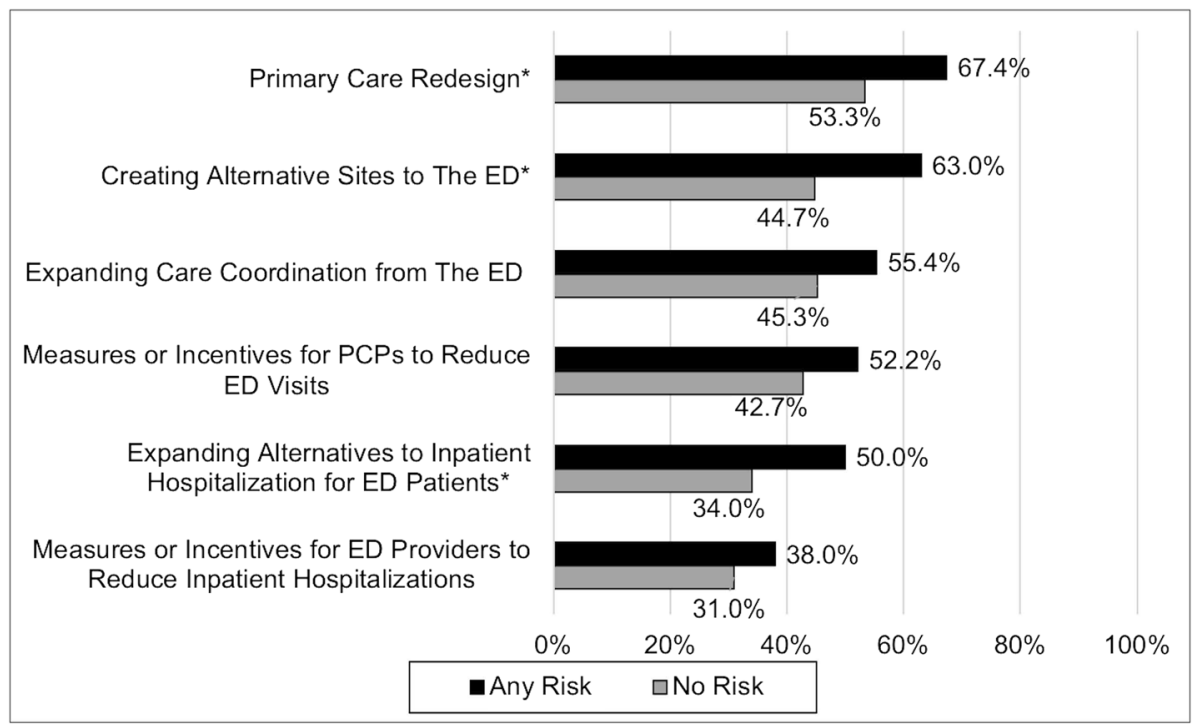

Fig. 2 Comparison of acute care priorities for ACOs with and without risk-bearing contracts. $* P<0.05$. 
Acknowledgements: We would like to acknowledge Kerstin Edwards, MPA, Research Manager at Leavitt Partners and Teresa Litton, MPH, Senior Policy Advisor at the National Association of ACOs, for their contributions to this work

Corresponding Author: Michelle P. Lin, MD, MPH, MS; Department of Emergency Medicine Icahn School of Medicine at Mount Sinai, New York, NY, USA (e-mail: michelle.lin@mountsinai.org).

Funding Information This project was funded by a Health Policy Research grant from the Emergency Medicine Foundation (Schuur, Lin). An abstract was presented at ACEP Scientific Assembly, Washington, DC, October 30, 2017.

\section{Compliance with Ethical Standards}

Conflict of Interest: Mr. Muhlestein is an employee of the for-profit Leavitt Partners, LLC. Dr. Carr serves as Director of the Emergency Care Coordination Center in the US Department of Health and Human Services; the views expressed here do not necessarily represent those of the government. He has also received payment for legal consulting from BGC. Dr. Wiler has received royalty payments from AgileMD and CareThrough. Dr. Schuur has received compensation for his work on the Scientific Advisory Board for UnitedHealth. All remaining authors declare that they do not have a conflict of interest.

\section{REFERENCES}

1. Lee MH, Schuur JD, Zink BJ. Owning the cost of emergency medicine: beyond 2\%. Ann Emerg Med. 2013;62(5):498-505.e3. https://doi.org/10. 1016/j.annemergmed.2013.03.029.

2. deLisle K, Litton T, Brennan A, Muhlestein D. The 2017 ACO Survey: What Do Current Trends Tell Us About The Future Of Accountable Care? Health Affairs Blog. October 4, 2017. Available at: https://www. healthaffairs.org/do/10.1377/hblog20171021.165999/full/ Accessed on 17 Apr 2018.

3. Whittaker W, Anselmi L, Kristensen SR, et al. Associations between extending access to primary care and emergency department visits: a difference-in-differences analysis. PLoS Med. 2016;13(9):e1002113. https://doi.org/10.1371/journal.pmed. 1002113.

4. David G, Gunnarsson C, Saynisch PA, Chawla R, Nigam S. Do patientcentered medical homes reduce emergency department visits? Health Serv Res. 2015;50(2):418-439. https://doi.org/10.1111/1475-6773.12218.

5. Raven MC, Kushel M, Ko MJ, Penko J, Bindman AB. The effectiveness of emergency department visit reduction programs: a systematic review. Ann Emerg Med. 2016;68(4):467-483.e15. https://doi.org/10.1016/j. annemergmed.2016.04.015.

6. Levinson, Daniel R. Office of the Inspector General. Medicare Shared Saving Program Accountable Care Organizations Have Shown Potential for Reducing Spending and Improving Quality. Department of Health and Human Services. OEI-02-15-00450 August, 2017. Available at: https:// oig.hhs.gov/oei/reports/oei-02-15-00450.asp Accessed on 17 Apr 2018. 